\title{
Chiral Symmetry of Double-Walled Carbon Nanotubes detected in First-principles Optical Absorption Spectra
}

\author{
Xiaoping Yang, ${ }^{1,2}$ Jiangwei Chen, ${ }^{1}$ Hui Jiang ${ }^{1}$ and Jinming Dong ${ }^{1}$ \\ ${ }^{1}$ National Laboratory of Solid State Microstructures, \\ and Department of Physics, Nanjing University, Nanjing 210093, P. R. China \\ ${ }^{2}$ Department of Physics, Huainan Normal University, \\ Huainan, Anhui Province 232001, P. R. China
}

(Dated: 2 March 2007)

\begin{abstract}
The linear polarizability absorption spectra of the double-walled carbon nanotubes (DWNTs) have been calculated by using the tight-binding (TB) model and sum-over-state (SOS) method, supplemented by the first principles CASTEP calculations. It is found that the chiral symmetries of both outer and inner tubes in the DWNTs can always be identified distinctly by the characteristic peaks in the absorption spectra of the DWNTs, no matter what kind of the outer tube is, offering a powerful experimental tool to measure precisely the chiral angle of the inner tube of a DWNT.
\end{abstract}

PACS numbers: PACS numbers: 78.67. Ch, 73.22. -f, 78.40. Ri 
In the past decade carbon nanotubes (CNTs) [1-3], both single-walled (SWNT) and multi-walled (MWNT) had been extensively investigated, which is motivated by their special electrical and mechanical properties as well as by their potential applications in future's nanostructured materials. Their unique electronic structures and mechanical properties are proved to be a rich source of new fundamental physics and also make them promising candidates as nanoscale quantum wires, single electron and field-effect transistors and sensors.

The SWNT is composed of a rolled up 2D-graphite sheet, and discovered first by Iijima's group in 1991. The carbon atoms on a SWNT are arranged on a helical line around its axis. The geometrical structure of the SWNT defined by a pair of intergers $(n, m)$ determines its radius and chirality, and so entirely its electronic structure, and optical property. It is known that the SWNTs with $n-m$ being a multiple of 3 are metallic, and all others are semiconducting [4].

The DWNT is the simplest type of MWNT, which has been made experimentally by many kinds of methods, and consists of two concentric cylindrical graphene layers. The nucleation of the inner tube should occur after the growth of the outer tube according to the 'yarmulke mechanism' [5], which means that the inner tube diameter should be determined by the outer tube one.

Early studies on DWNTs focused theoretically on their electronic structure, stability, etc. [6-9]. A DWNT can be composed of a pair of inner and outer constituent layers with any chiralities, making different kinds of DWNTs, such as metal-metal, metal-semiconducting, or semiconducting-semiconducting nanotubes. It was shown that the band structure of a DWNT depends on the combined configurations of the inner and outer tubes [7], but their stability depends only on their interlayer spacing [6].

On the other hand, it is of fundamental and practical importance to characterize the detailed geometrical structure of a DWNT because it has a close relationship with the electronic, mechanical and optical properties of the DWNT. It is in practice, however, a very difficult task to determine the geometrical structure of a DWNT, especially its inner tube. Usually, both scattering tunneling spectroscope (STS) and scattering tunneling microscope (STM) can be used to roughly measure the outer tube radius, and so approximate radius of the inner tube. However, the chiral angle of the inner tube can not be known by this kind of measurements. The Resonant Raman Scattering and selected area electron diffraction (SAED) measurements combined with the theoretical simulations have also been used to determine the chiral indices of SWNTs, and are in development to get information about those of the inner tubes in DWNTs $[10,11]$.

It is well known that the optical absorption is a fundamental property of a solid and can be easily and exactly measured experimentally. In general, the optical property can be well described by the electronic energy band structure of the material, which has a close relation with its geometrical structure, especially in the nanostructured materials. In this paper, we investigate how the chiral indices of the inner tubes of DWNTs can be identified unambiguously by an analysis of the optical spectra of the DWNTs.

We firstly use a TB model with one $\pi$-orbital per carbon atom to get the electronic structures of the DWNTs, which has been successfully applied to describe the SWNT, DWNT, and MWNT [12-14], and then use the SOS approach to calculate the linear absorption spectra $[15,16]$ of DWNTs with finite lengths in real space.

The TB Hamiltonian is given by [12-14]

$$
H=\gamma_{0} \sum_{i, j} c_{j}^{\dagger} c_{i}-W \sum_{i^{\prime}, j^{\prime}} \cos \left(\theta_{i^{\prime} j^{\prime}}\right) e^{\left(a-d_{i^{\prime} j^{\prime}}\right) / \delta} c_{j^{\prime}}^{\dagger} c_{i^{\prime}},
$$


where $\gamma_{0}(=-2.75 \mathrm{eV})$ is the intra-layer hopping parameter between the nearest neighbor sites, $i$ and $j$, and $W$ is the inter-wall interactions strength between interlayer sites, $i^{\prime}$ and $j^{\prime}$, with a distance of $d_{i^{\prime} j^{\prime}}$ and the cutoff of $d_{i^{\prime} j^{\prime}}>3.9 \AA$. Here $\theta_{i^{\prime} j^{\prime}}$ is the angle between two $\pi$-orbitals on the sites $i^{\prime}$ and $j^{\prime}$, and $c_{i}$ is the annihilation operator of an electron on site $i$. $a(=3.34 \AA)$ is the distance between two carbon walls, and $\delta=0.45 \AA$. As well known, the parameters used in the TB model are usually obtained by fitting the TB calculation results with those of the ab initio calculations, or the experimental data. Estimation based on ab initio calculations of $W$ gives $W\left(=\gamma_{0} / 8\right)$. The weak inter-layer interaction is caused by the larger inter-layer distance of $3.4 \AA$, compared with intra-layer bond length of $1.42 \AA$.

Within the independent electron approximation and SOS approach, the linear polarizability $\alpha(\omega)$ is expressed as [15]

$$
\alpha(\omega)=2 \sum_{\substack{n \in \text { occ } \\ p \in \text { unocc }}} \mu_{n p} \mu_{p n}\left(\frac{1}{\varepsilon_{p n}-\omega}+\frac{1}{\varepsilon_{p n}+\omega}\right)
$$

In the above formula, $\varepsilon_{n p}=\varepsilon_{n}-\varepsilon_{p}$, and $\mu_{n m}$ is the dipole transition matrix elements between the one-electron state $Z_{n, s}$ and $Z_{m, s}$, which is given by

$$
\left\langle n\left|\mu_{\alpha}\right| m\right\rangle=\sum_{j, s} Z_{n, s}^{*}(j)\left(-e r_{j}\right) Z_{m, s}(j)
$$

Here, $r_{j}$ is the coordinate of the $j_{t h}$ atom. The linear polarizability absorption spectra is the imaginary part of the linear polarizability $\alpha(\omega)$.

Then, we have also made the first-principle calculations on the polarized absorption spectra of the DWNT by the CASTEP code [17], which is compared with the results obtained by the TB method.

The nanotubes are usually treated as infinite periodic one dimensional crystals in the theoretical studies. But in practical experiments, the nanotubes have finite lengths. So, it is interesting to study physical properties of the carbon nanotubes with finite lengths, which has big effects on their electronic structures and corresponding optical responses $[16,18]$. For example, the finite length $(n, m)$ SWNTs with $n-m$ being not multiples of 3 will have smaller linear polarizability $\alpha(\omega)[16]$.

In our calculations, the nanotube is cut perpendicular to its axis, making it to have two open ends. Taking into account the computational time and cost, we select the calculated CNT length of up to $172.2 \AA$, which is equivalent to that of 70 periods of the armchair tube $(12,12)$. Correspondingly, the calculated energy region for the optical spectrum is restricted in the $0.4 \sim 4 \mathrm{eV}$. The interlayer distance of DWNTs is usually taken as $3.4 \AA$.

Firstly, we choose two kinds of SWNT as the possible outer tube of the DWNTs: armchair tube $(12,12)$ (radius, $r=8.136 \AA)$ and chiral semiconductor tube $(13,11)(r=8.134$ $\AA)$. Their absorption spectra $\alpha_{\|}(\omega)$ polarized to tube direction are calculated in the TB approximation and shown in Figs. 1(a), 1(b), respectively. And their corresponding densities of states (DOSs) are shown in Figs. 1(c) and 1(d), respectively. It is seen that the characteristic absorption peaks labeled by symblo 'o' lie at 1.45, 2.8 and $3.9 \mathrm{eV}$ for $(12,12)$ tube, and at $0.5,1.0,1.9,2.3,3.2$ and $3.5 \mathrm{eV}$ for $(13,11)$ tube, which are contributed by the $1^{\text {st }}, 2^{\text {nd }}, 3^{\text {rd }}$, etc, pair of Van Hove Singularity (VHS) DOS peaks. For example, it can be clearly seen that the distances between the $1^{\text {st }}$ pair of VHS DOS peaks for $(12,12)$, and $(13,11)$ tubes are about $1.45 \mathrm{eV}$, and $0.5 \mathrm{eV}$, respectively, which correspond exactly to the 
positions of two $1^{\text {st }}$ absorption peaks for both SWNTs [seen from Figs. 1(a) and 1(b)]. The characteristic absorption peaks will become more evident when the calculated CNT length is taken to be longer.

For these two outer tubes, different possible inner tubes are chosen and studied as follows.

The inner tube is armchair $(7,7)$ tube $(r=4.746 \AA)$. In this case, two absorption spectra $\alpha_{\|}(\omega)$ for two DWNTs, commensurate $(7,7) @(12,12)$, and incommensurate $(7,7) @(13,11)$, are calculated in the TB approximation and shown in Fig. 2, in which are also shown the corresponding results for pure $(7,7)$ tube. In order to define more directly the characteristic absorption peaks of the inner tube, we also show the joint density of states (JDOS) in Fig. 2 (a) for the vertical transition between the same band index. The only one sharp peak at $2.4 \mathrm{eV}$ in the JDOS of the inner $(7,7)$ tube comes from the $1^{\text {st }}$ pair of the VHS peaks in its DOS, which induces one sharp absorption peak at $2.4 \mathrm{eV}$, shown in Fig. 2(b) and labeled by a symbol ' $i$ '. It is seen by comparing Fig. 1 and Fig. 2 that all the characteristic absorption peaks of both outer and inner tubes can be identified distinctly in the absorption spectra [Figs. 2(c) and 2(d)] of the DWNTs with an allowable shift of about $\pm 0.1 \mathrm{eV}$, which is caused by the splitting of the degenerated bands, induced by the weak interlayer coupling in the DWNT. For example, the $1^{\text {st }}$ absorption peak of the inner $(7,7)$ tube can be found clearly in the absorption spectra of both DWNTs [Figs. 2(c) and 2(d)] although it happens to overlap with a peak of the outer tube $(13,11)$ at $2.4 \mathrm{eV}$ in the DWNT $(7,7) @(13,11)$.

We can also choose a chiral metal tube $(11,2)(r=4.746 \AA)$ as the inner tube, whose period is equal to seven times of that for $(12,12)$ tube, and incommensurate to that of tube $(13,11)$. The calculated JDOS of $\operatorname{SWNT}(11,2)$ is shown in Fig. 3(a), and the absorption spectra $\alpha_{\|}(\omega)$ for pure tube $(11,2)$, the commensurate DWNT $(11,2) @(12$, 12), and incommensurate DWNT $(11,2) @(13,11)$ are given in Figs. 3(b), 3(c) and 3(d), respectively. Since each VHS peak of the inner chiral metal tube $(11,2)$ is now split into two with much different heights, there appear two sharp JDOS peaks in Fig. 3(a), which induce two big absorption peaks at 2.3 and $2.6 \mathrm{eV}$, shown in Fig. 3(b). Both of them can also be identified distinctly in the absorption spectra of the two DWNTs [Figs. 3(c) and $3(\mathrm{~d})]$ with the same shift of about $\pm 0.1 \mathrm{eV}$. In the same time, the characteristic absorption peaks of outer tubes $(12,12)$ and $(13,11)$ can be found clearly in the absorption spectra of two DWNTs. So, it is obvious that the chiral indices of a chiral or achiral inner metal tube can be identified well by the absorption spectra of corresponding DWNTs no matter the outer tube is metal or semiconductor.

Above, both metal tubes, one $(7,7)$ and another $(11,2)$ are chosen as the inner tube of the DWNTs. Now, we choose a chiral semiconducting tube $(8,6)(r=4.762 \AA)$ as the inner tube. Its JDOS is given in Fig. 4(a). The calculated absorption spectra $\alpha_{\|}(\omega)$ for pure $(8,6)$ tube, the incommensurate DWNTs $(8,6) @(12,12)$ and $(8,6) @(13,11)$ are shown in Figs. 4(b), 4(c), and 4(d), respectively. Now, the more JDOS peaks of the inner $(8,6)$ tube appear in the energy window of 0 to $4 \mathrm{eV}$, producing thus more characteristic absorption peaks at $0.85,1.65,3.2$ and $3.7 \mathrm{eV}$ in Fig. 4(b). Even so, it is clearly seen from Figs. $4(\mathrm{c})$ and $4(\mathrm{~d})$ that these four absorption peaks of the inner tube $(8,6)$ can always be well identified although again the $3^{\text {rd }}$ peak coincides with one absorption peak of outer tube $(13$, 11) in Fig. 4(d).

From the above results, it is known that no matter the inner tube is metal or semiconductor, achiral or chiral, its characteristic absorption peaks can always be identified clearly from the absorption spectra of the corresponding DWNTs, which is independent of selections of the outer tubes, indicating the inter-tube coupling has only minor effects on the absorption 
spectra of a DWNT. So, we can conclude that it is possible experimentally to deduce the chiral indices of the inner tube for any DWNTs by comparing their absorption spectra with those different types of SWNTs.

Finally, in order to check correctness of our TB calculations, we have also made the first-principle calculations in the framework of local density approximation (LDA) on the polarized absorption spectra $\alpha_{\|}(\omega)$ of the DWNT $(7,7) @(12,12)$ and related SWNTs $(7$, $7)$ and $(12,12)$ by the CASTEP code $[17]$, in which the exchange-correlation energy of the Ceperley and Alder form [19] was used. The ion-electron interaction is modeled by ultrasoft local pseudopotentials of the Vanderbilt form [20] for the carbon atoms with maximum plane wave cut-off energy of $240 \mathrm{eV}$. In the calculation, a supercell geometry [21] is used in which the tubes are aligned in a hexagonal array with an adjacent inter-tube distance of 28 $\AA$, being larger enough to prevent the tube-tube interactions. The space group is $P_{1}$ for the computational models. The Monkhorst-Pack scheme [22] with a distance of $0.03 / \AA$ between points is used for the sampling in reciprocal space. The calculated results are given in Fig. 5. The absorption peaks of SWNT $(7,7)$ are found to be at $2.45,3.44$ and $3.84 \mathrm{eV}$, and those for SWNT $(12,12)$ are at 1.44, 2.09, 2.54, 2.79, 3.19 and $3.94 \mathrm{eV}$. And those peaks for DWNT $(7,7) @(12,12)$ lie at 1.38, 1.87, 2.04, 2.45, 2.74, 3.10, 3.37, 3.53, 3.78 and 4.03 $\mathrm{eV}$. By comparison, we find that the absorption peaks of both the outer and inner tube can be all identified distinctly in the absorption spectra of the DWNT with an allowable shift of about $\pm 0.1 \mathrm{eV}$, which comes also from the interlayer interaction of the DWNT.

In addition, comparing Figs. 5(a) and 5(b) with Fig. 1(a) and Fig. 2(b), we know that the characteristic absorption peaks of pure $(7,7)$ and $(12,12)$ tubes obtained by the TB model and SOS method can all be found in their absorption spectra got from the firstprinciples calculations, which lie at $2.45 \mathrm{eV}$ for $(7,7)$ tube, and at $1.44,2.79$, and $3.94 \mathrm{eV}$ for $(12,12)$ tube with an allowable shift of about $\pm 0.05 \mathrm{eV}$. So, we conclude that the obtained results by the TB model and SOS method are much closer to those by the first-principles method, especially for the absorption peak positions, demonstrating that both of the TB model and SOS method are suitable for the calculations of the linear optical absorptions for the DWNTs and SWNTs.

In summary, it is shown by the numerical calculations that the optical responses can be used to identify clearly the chiral symmetries of the outer and inner tubes in the DWNTs, based upon which a powerful experimental tool can be proposed for measuring unambiguously the chiral angle of the inner tube of a DWNT.

This work was supported by the Natural Science Foundation of China under Grant No.10074026, No. 90103038 and No. A040108. The authors acknowledge also support from a Grant for State Key Program of China through Grant No.1998061407.

1 S. Iijima, Nature (London) 354, 56 (1991).

2 T.W. Ebbesen, P.M. Ajayan, Nature (London) 358, 220 (1992).

3 J. Kong, H.T. Soh, A.M. Cassell, C.F. Quate, H. Dai, Nature (London) 395, 878 (1998).

4 R. Saito, G. Dresselhaus, and M.S. Dresselhaus, Physical Properties of Carbon Nanotubes (Imperial College Press, London, 1998).

5 H.J. Dai, A.G. Rinzler, P. Nikolaev, A. Thess, D.T. Colbert, R.E. Smalley, Chem. Phys. Lett. 260, 471 (1996). 
6 R. Saito, R. Matsuo, T. Kimura, G. Dresselhaus, M.S. Dresselhaus, Chem. Phys. Lett. 348, 187 (2001).

7 K. Tanaka, H. Aoki, H. Ago, T. Yamabe, K. Okahara, Carbon 35, 121 (1997).

8 Y.K. Kwon, D. Tomanek, Phys. Rev. B 58, R16001 (1998).

9 M. Buongiorno Nardelli, C. Brabec, A. Maiti, C. Roland, J. Bernholc, Phys. Rev. Lett. 80, 313 (1998).

10 M. Kociak, K. Suenaga, K. Hirahara, Y. Saito, T. Nakahira, and S. Iijima, Phys. Rev. Lett. 89, 155501 (2002); S. Bandow, G. Chen, G.U. Sumanasekera, R. Gupta, M. Yudasaka, S. Iijima, and P.C. Eklund, Phys. Rev. B 66, 075416 (2002); S. Bandow, M. Takizawa, K. Hirahara, M. Yudasaka, and S. Iijima, Chem. Phys. Lett. 337, 48 (2001).

11 Zhenping Zhou, Lijie Ci, Xihua Chen, Dongsheng Tang, Xiaoqin Yan, Dongfang Liu, Yingxin Liang, Huajun Yuan, Weiya Zhou, Gang Wang, and Sishen Xie, Carbon 41, 337 (2003); Lijie Ci et al., Chem. Phys. Lett. 359, 63 (2002).

12 K.H. Ahn, Y.H. Kim, J.Wiersig, and K.J. Chang, Phy. Rev. Lett. 90, 026601 (2003).

13 R. Saito, G. Dresselhaus, and M.S. Dresselhaus, J. Appl. Phys. 73, 494 (1993); J.C. Charlier and J.P. Michenaud, Phys. Rev. Lett. 70, 1858 (1993).

14 S. Roche, F. Triozon, A. Rubio, D. Mayou, Phys. Rev. B 64, 121401 (2001).

15 J. Yu and W. P. Su, Phys. Rev. B 44, 13315 (1991); Jinming Dong, J. Jiang, J. Yu, Z.D. Wang, and D.Y. Xing, Phys. Rev. B 52, 9066 (1995).

16 X. Wan, and J. Dong, Phy. Rev. B 58, 6756 (1998).

17 CASTEP is an ab initio program with plane-wave pseudopotential package distributed by MSI.

18 J. Jiang, J. Dong, and D.Y. Xing, Phy. Rev. B 65, 245418 (2002).

19 D.M. Ceperley, B. J. Alder, Phys. Rev. Lett. 45, 566 (1980).

20 A.M. Rappe, K.M. Rabe, E. Kaxiras, J.D. Joannopoulos, Phys. Rev. B 41, 1227 (1990).

21 X. Blase, Lorin X. Benedict, Eric L. Shirley, Steven G. Louie, Phys. Rev. Lett. 72, 1878 (1994).

22 H.J. Monkhorst, J.D. Pack, Phys. Rev. B 13, 5188 (1976). 

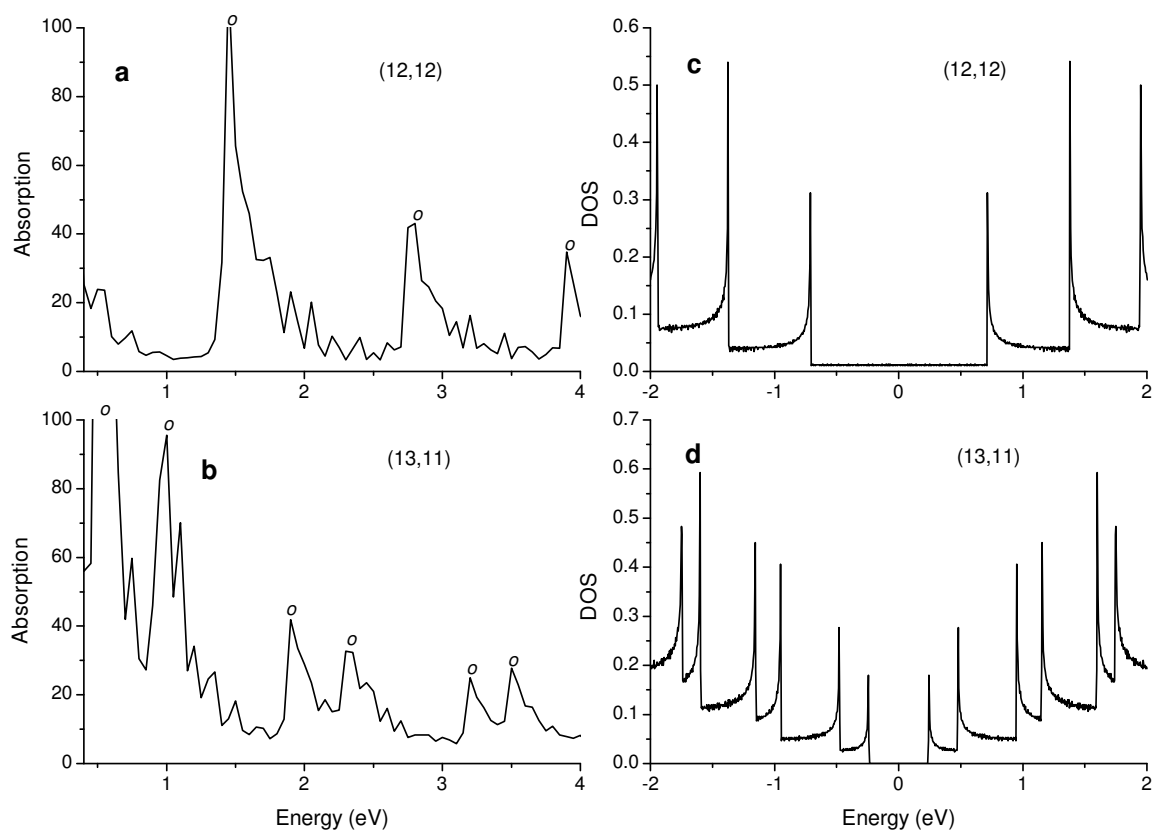

FIG. 1: The absorption spectra $\alpha_{\|}(\omega)$ (in $\AA^{3}$ ) for (a) $(12,12)$ tube , (b) $(13,11)$ tube and corresponding density of states for $(\mathrm{c})(12,12)$ tube, (d) $(13,11)$ tube. The characteristic absorption peaks of the outer and inner tubes are labeled by the symbol ' $o$ ' and ' $i$ ', those are the same in the other figures of this paper. 

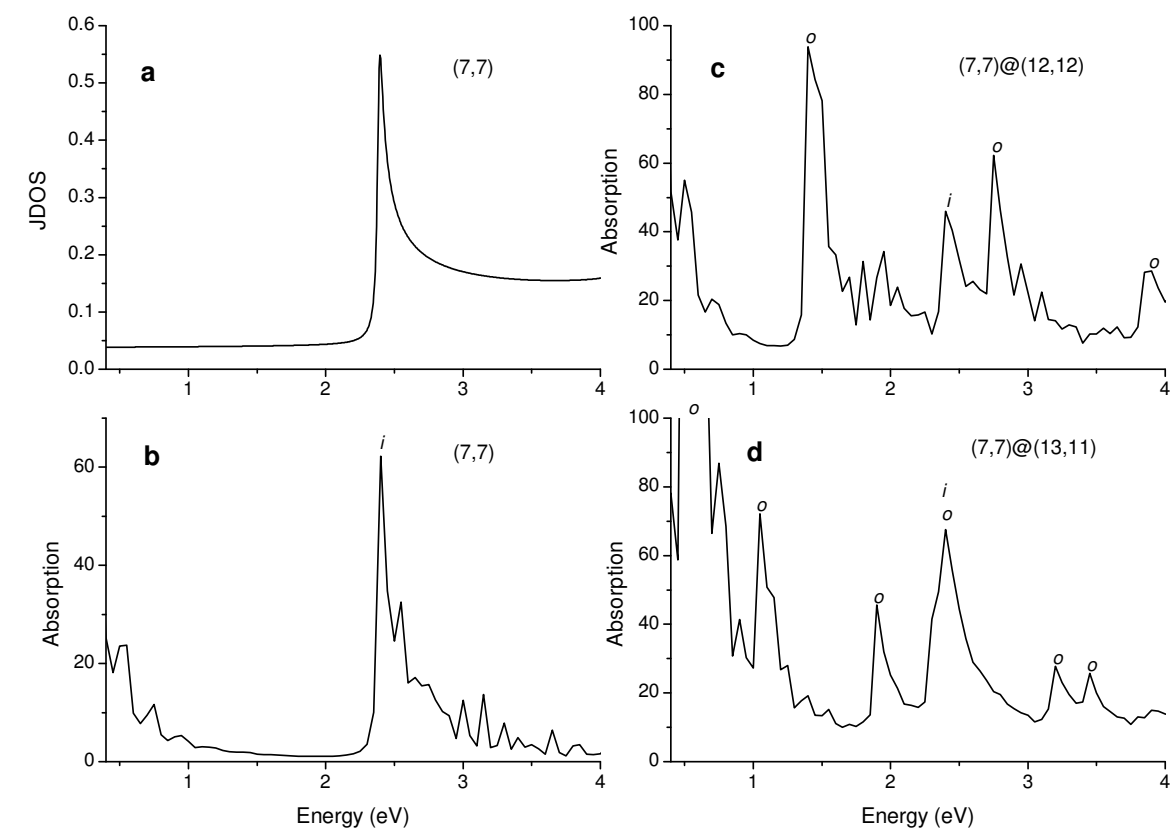

FIG. 2: (a) JDOS of $(7,7)$ tube. The absorption spectra $\alpha_{\|}(\omega)$ (in $\AA^{3}$ ) for (b) pure $(7,7)$ tube, (c) DWNT $(7,7) @(12,12)$, and (d) DWNT $(7,7) @(13,11)$.
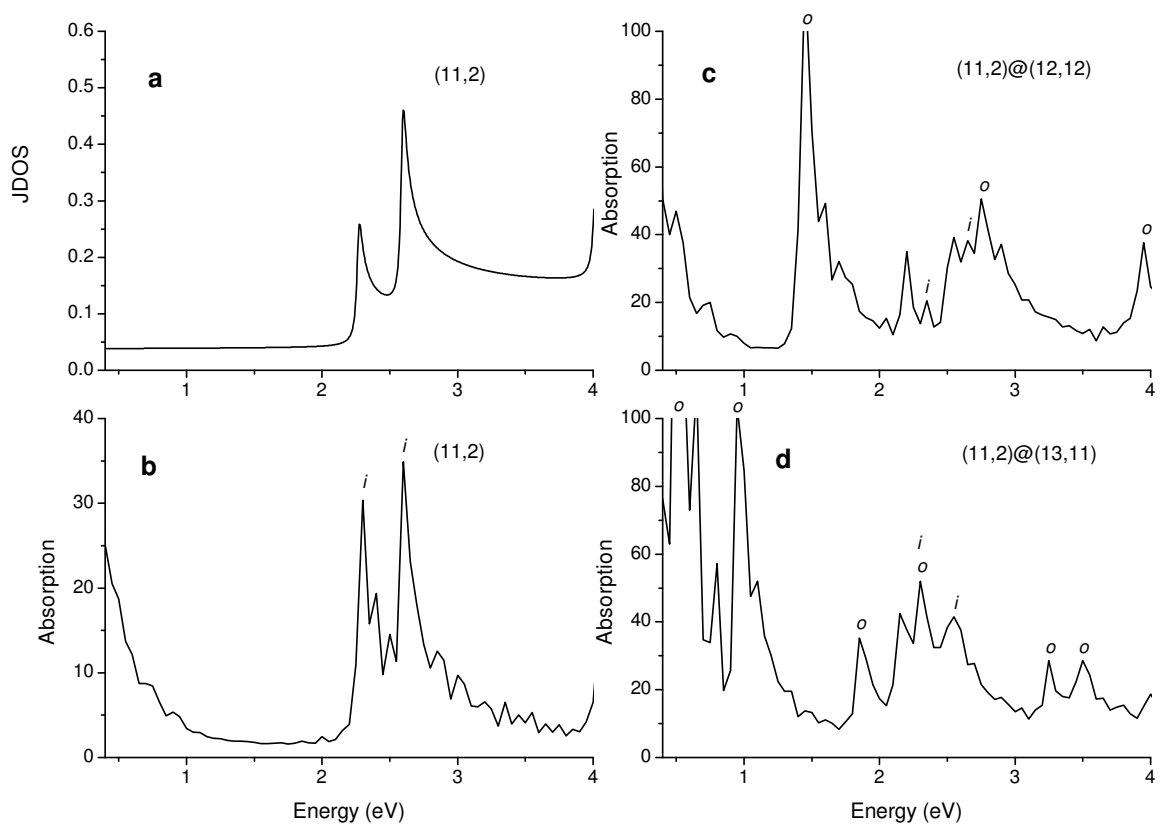

FIG. 3: (a) JDOS of pure $(11,2)$ tube. The absorption spectra $\alpha_{\|}(\omega)$ (in $\AA^{3}$ ) for (b) pure $(11,2)$ tube, (c) DWNT $(11,2) @(12,12)$, (d) DWNT $(11,2) @(13,11)$. 

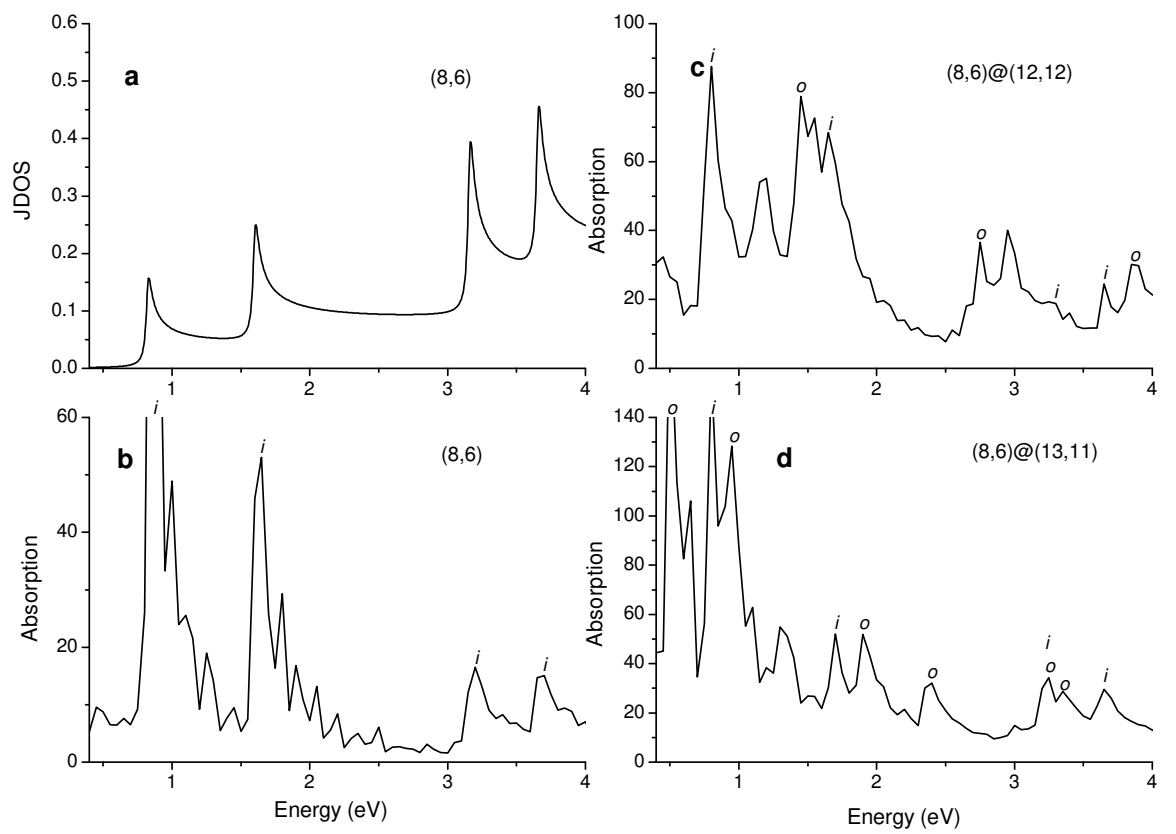

FIG. 4: (a) JDOS of pure $(8,6)$ tube. The absorption spectra $\alpha_{\|}(\omega)$ (in $\left.\AA^{3}\right)(b)$ pure $(8,6)$ tube, (c) DWNT $(8,6) @(12,12)$, and (d) DWNT $(8,6) @(13,11)$. 


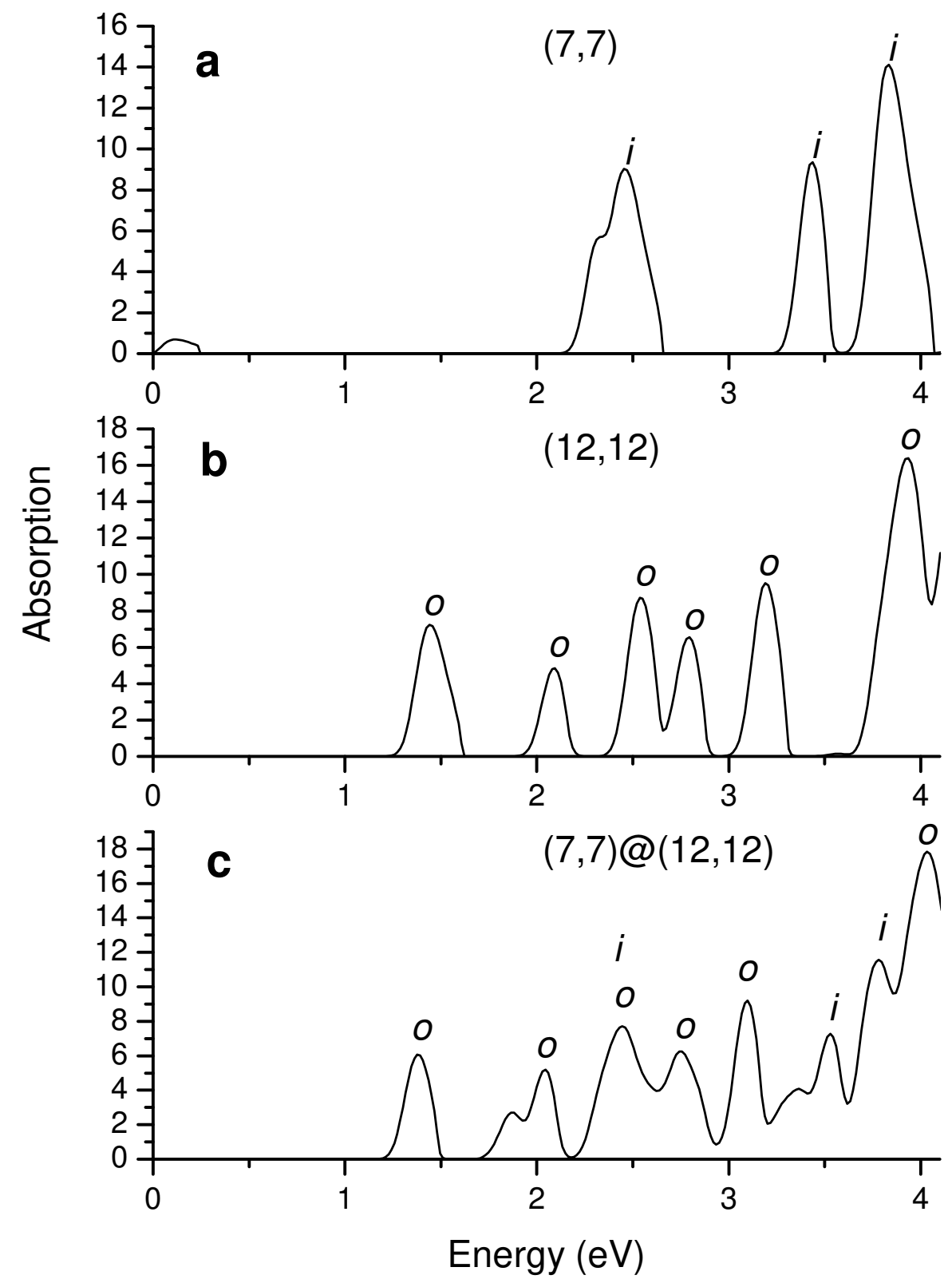

FIG. 5: The absorption spectra $\alpha_{\|}(\omega)$ (in $\AA^{3}$ ) for (a) pure $(7,7)$ tube, (b) pure $(12,12)$ tube, and (c) DWNT $(7,7) @(12,12)$ obtained by the first-principles calculation. 